University of Montana

ScholarWorks at University of Montana

Public and Community Health Sciences Faculty

Publications

Public and Community Health Sciences

2006

\title{
Adherence to Principles of Motivational Interviewing and Client Within-Session Behavior
}

Delwyn Catley

Kari Jo Harris

The University of Montana

Matthew S. Mayo

Sandra Hall

Kolawole S. Okuyemi

See next page for additional authors

Follow this and additional works at: https://scholarworks.umt.edu/pchs_pubs

Part of the Public Health Commons

Let us know how access to this document benefits you.

\section{Recommended Citation}

Catley, Delwyn; Harris, Kari Jo; Mayo, Matthew S.; Hall, Sandra; Okuyemi, Kolawole S.; Boardman, Thuy; and Ahluwalia, Jasjit S., "Adherence to Principles of Motivational Interviewing and Client Within-Session Behavior" (2006). Public and Community Health Sciences Faculty Publications. 23.

https://scholarworks.umt.edu/pchs_pubs/23

This Article is brought to you for free and open access by the Public and Community Health Sciences at ScholarWorks at University of Montana. It has been accepted for inclusion in Public and Community Health Sciences Faculty Publications by an authorized administrator of ScholarWorks at University of Montana. For more information, please contact scholarworks@mso.umt.edu. 


\section{Authors}

Delwyn Catley, Kari Jo Harris, Matthew S. Mayo, Sandra Hall, Kolawole S. Okuyemi, Thuy Boardman, and Jasjit S. Ahluwalia 
Behavioural and Cognitive Psychotherapy, 2006, 34, 43-56

Printed in the United Kingdom doi:10.1017/S1352465805002432

\title{
Adherence to Principles of Motivational Interviewing and Client Within-Session Behavior
}

\author{
Delwyn Catley \\ University of Missouri - Kansas City, Kansas City, USA
}

Kari Jo Harris

The University of Montana, Missoula, USA

\author{
Matthew S. Mayo, Sandra Hall, Kolawole S. Okuyemi, Thuy Boardman \\ and Jasjit S. Ahluwalia \\ University of Kansas School of Medicine, Kansas City, USA
}

\begin{abstract}
The purpose of this study was to examine whether counselor adherence to Motivational Interviewing (MI) principles was associated with more productive within-session client behavior in a smoking cessation trial for African American smokers. For these analyses 89 baseline counseling sessions of the trial were audiotaped and coded using the Motivational Interviewing Skill Code (MISC). Counselor adherence indicators included a global subjective rating of MI adherence and the frequency of MI-consistent and MI-inconsistent counselor behaviors described in the MISC. Indicators of productive client behaviors included global subjective ratings of within-session client functioning and counselor-client interaction, as well as the frequency of statements by the client favorable toward changing behavior ("change talk") and resistant regarding changing behavior ("resist-change talk"). Results provided support for the principles of MI. Counselor adherence indexed by both the global subjective rating and MI-consistent behavior frequency was significantly positively associated with global ratings of within-session client functioning and counselor-client interaction, as well as more change talk.
\end{abstract}

Keywords: Motivational Interviewing, smoking, counseling, African American.

Motivational Interviewing (MI) is a therapeutic method designed to facilitate behavior change. Initially developed in the field of addictions, particularly in alcohol addiction treatment, it is increasingly being applied to a wider variety of problems including health behaviors and other addictive behaviors such as smoking (Resnicow, Diiorio et al., 2002). The "spirit", theory,

Reprint requests to Delwyn Catley, Department of Psychology, University of Missouri - Kansas City, 5100 Rockhill Road, Kansas City, MO 64110-2499, USA. E-mail: catleyd@umkc.edu 
and practical application of MI methods have been described in detail (Miller and Rollnick, 1991, 2002). In brief, MI has been defined as a client-centered, directive method for enhancing intrinsic motivation to change by exploring and resolving ambivalence. The emphasis is on the use of a collaborative style in which motivation is elicited rather than imposed, and the individual's autonomy regarding change is affirmed. There are four general principles: to express empathy, to develop discrepancy between current behavior and important goals or values, to "roll with" or avoid struggling against resistance, and to support self-efficacy for change. Specific methods recommended include using open-ended questions, listening reflectively, affirming, and summarizing.

While randomized controlled trials have generally supported the efficacy of MI, most of the studies lacked verification of fidelity to MI to ensure that MI was in fact delivered (e.g., Brown and Miller, 1993; Gentilello et al., 1999; Handmaker, Miller and Manicke, 1999; for a review see Burke, Arkowitz and Dunn, 2002). Related to this is the lack of research examining which principles of MI actually account for positive therapeutic outcomes. As interest in using MI rapidly increases, it is important to deepen understanding of how MI works to ensure effective training and treatment. We recently conducted a smoking cessation intervention trial in which all participants received counseling from counselors trained in MI principles. This offered a good opportunity to examine the extent to which counselor adherence to MI principles predicted productive within-session client behaviors.

A coding scheme known as the Motivational Interviewing Skills Code (MISC; Miller and Mount, 2001, Moyers, Martin, Catley, Harris and Ahluwalia, 2003; Tappin et al., 2000) has been developed that operationalizes the principles described by Miller and Rollnick (1991, 2002) in terms of specific therapist behaviors. Counselor adherence to MI principles is indexed in global terms regarding the degree of the counselor's acceptance of the client, spirit of egalitarianism, empathy, genuineness, warmth and overall "MI spirit". In addition, specific behavior counts are made of prescribed and proscribed behaviors. MI-consistent counselor statements include advising only with permission, affirming the client, emphasizing client control, using open-ended questions, using reflective statements, using reframing, and making statements of support. MI-inconsistent behaviors include advising without permission, direct confrontation, raising concern without permission, and warning. Experts in MI have been rated very highly in adherence to each of these principles using this coding scheme, suggesting that it can be useful as a measure of the extent to which MI is being delivered (Miller and Mount, 2001).

Of particular interest in this study was whether counselor adherence to MI principles would be associated with a particular productive client behavior known as "change talk" (Miller and Rollnick, 2002). Change talk is thought to increase the likelihood of subsequent behavior change and is therefore a theoretically important indicator of "good MI". For example, a recent study of MI treatment of drug abusers showed an association between statements of commitment to change and therapeutic outcomes (Amrhein, Miller, Yahne, Palmer and Fulcher, 2003). Change talk includes recognizing disadvantages of the status-quo (e.g. disadvantages of continuing to smoke), stating advantages of changing, expressing optimism for change, and expressing intention to change. Related to change talk is the converse response in which participants deny the need for or resist the notion of change. Drawing from the terminology of the MISC system we refer to the former as "change talk" and the latter as "resist-change talk". 
Studies of addiction treatment have indicated that an empathic style is associated with positive client outcomes (Valle, 1981; Miller, Taylor and West, 1980; Miller and Baca, 1983). Two previous experimental studies have also examined the effects of counselor behaviors prescribed and proscribed by MI principles. A study by Patterson and Forgatch (1985) that was unrelated to MI experimentally manipulated therapist behaviors during parent training and found that "teaching" and "confronting" produced significant increases in negative client behavior (such as interrupting and not tracking), whereas therapist "supporting" and "facilitating" behavior reduced negative client responses. Miller, Benefield and Tonigan (1993) examined the effects of a client-centered MI style in comparison to a directiveconfrontational style for problem drinkers on within-session and end of treatment/follow-up drinking outcomes. Although there were no significant effects of the assigned counseling style (i.e. experimental group) on drinking behaviors, the frequency of counselor confrontation (i.e. challenging, disagreeing, disputing, etc) was correlated with greater drinking behavior at follow-up. In addition, in the MI-consistent condition there was a lower frequency of negative client responses such as arguing, denying problems, and interrupting and a higher frequency of clients acknowledging problems. The frequency of negative client responses was in turn related to greater drinking at the 12-month follow-up.

Although these studies are generally supportive of MI principles, they either did not involve an MI-based intervention (Miller and Baca, 1983; Miller et al., 1980; Patterson and Forgatch, 1985; Valle, 1981) or lacked a coding system such as the MISC, which is specifically designed to assess therapist behavior using an MI framework (Miller et al., 1993). In addition, no studies have examined the use of MI-consistent principles and within-session client behavior in smoking cessation.

The purpose of this study was therefore to examine whether counselor adherence to MI principles was predictive of more productive within-session client behavior including more change talk and less resistance. Productive within-session behavior was indexed by subjective observer ratings of the overall functioning of the client (e.g. cooperating, being engaged) and the quality of the client-therapist interactions (i.e. collaboration and benefit) during sessions. Client change talk and resistance were assessed with observer frequency counts of change talk and resist-change talk statements. Based on previous studies and the principles articulated by Miller and Rollnick (2002), we hypothesized that greater MI adherence would be associated with better client within-session functioning, more positive counselor-client interactions, more change talk, and less resist-change talk.

In addition to these questions we were also interested in which specific aspects of MI adherence might be important in predicting the various indicators of within-session client behavior. Miller and Rollnick (1991, 2002) have not specified any particular MI-consistent or inconsistent behaviors as critical or most important, but those who have adapted MI for brief intervention often focus on particular principles. For example, some researchers have developed protocols that focus on eliciting change talk and developing discrepancy between current behavior and core values the client holds (e.g. Resnicow, Jackson et al., 2002; for a review see Wagner and Sanchez, 2002). In our training of MI counselors we strongly emphasize the use of reflective listening as a critical ingredient. Miller and Rollnick (2002) have also emphasized that technique is not a substitute for "MI spirit". To begin inquiry into this area, we conducted additional analyses to determine which of the individual counselor behaviors were significantly associated with productive client behavior. 


\begin{abstract}
Method
This study examined counseling provided as part of a double-blind, placebo controlled trial of bupropion hydrochloride as an aid to smoking cessation among African Americans. Study methods and outcomes are detailed elsewhere (Ahluwalia, Harris, Catley, Okuyemi and Mayo, 2002). In brief, 1,498 smokers in a mid-western city were screened and 981 were eligible and invited to participate. Persons eligible for the clinical trial described themselves as African American or black, were at least 18 years of age, smoked at least 10 cigarettes per day, were interested in quitting in the next 30 days, spoke English, and had a home address and working telephone. Participants were excluded if they had a medical contraindication for bupropion, were pregnant, currently used psychoactive medication, used other forms of tobacco or nicotine replacement in the past 30 days, were in drug treatment during the past 6 months, or were being treated for depression.

The 600 African Americans who enrolled were randomly assigned to receive $300 \mathrm{mg}$ of bupropion or placebo for 7 weeks and were followed for a total of 27 weeks. Participants in both the treatment and placebo groups received seven individual counseling sessions using Motivational Interviewing (MI). At the first visit, participants were provided with medication (or placebo), instructed to quit smoking in 7 days, and received approximately 30 minutes of counseling. For this supplementary study, the last 89 of these 600 baseline counseling sessions were tape-recorded and the first 20 minutes of counseling coded. Only the final 89 sessions were recorded because this supplementary study was proposed and funded subsequent to the start of the main study. All participants had the study explained to them and signed informed consent prior to tape-recording. The Human Subjects Committee of the University of Kansas Medical Center reviewed and approved the entire study protocol.

Because we only recorded the baseline sessions (out of a total of 7 sessions) of a small subset of participants (89 out of 600), and because the same counselor did not always work with a participant throughout, we were unable to effectively examine the effect of MI adherence on smoking cessation. The advantage of analyzing process variables from baseline sessions was that group assignment (drug vs. placebo) and attendance at MI sessions did not confound our analyses.
\end{abstract}

\title{
Participants
}

The final 89 people enrolled for the clinical trial comprised the initial sample of "clients" for the current study. Three audiotapes were unusable due to recording problems, resulting in a final sample of 86. On average, participants were 44 years old, predominantly female $(64 \%)$, and were relatively heavy smokers (mean cigarettes per day was 17). A little less than half of the participants $(41 \%)$ were married or living with a partner and a substantial proportion were of relatively low income (45\% less than $\$ 1800$ family income per month).

\section{Counselors}

Sessions were conducted by one of four African American Masters level counselors who had received training in MI. Counselors' training included attendance at an introductory workshop on MI as well as project-specific training sessions conducted by a study consultant 
with expertise in using MI in research interventions. Counselors were trained to follow a session outline developed for the study, which included exploring ambivalence about quitting, preparing to quit, problem-solving difficult situations, and problem-solving adherence with the medication. However, because MI was not a focus of the original study, counselors were not trained to meet any standard criterion of MI competency and did not receive ongoing supervision or verification of their fidelity to MI principles. Of the 86 total sessions coded, counselors contributed $31,29,15$, and 11 sessions, respectively.

\section{Coding}

All 86 audio-taped sessions were independently coded using the Motivational Interviewing Skills Code (MISC) by researchers who developed the MISC (Moyers et al., 2003). The MISC was developed to assess Motivational Interviewing during clinical interactions in which a specific behavior change is desirable (Miller and Mount, 2001). The MISC evaluates therapistclient interactions to provide quantifiable indices of the quality of Motivational Interviewing counseling delivered. After all 86 tapes had been coded, a convenience sample of 50 tapes was re-coded by a second coder who was blind to previous ratings. The interrater reliability achieved using the MISC on this data set has been described in detail elsewhere (Moyers et al., 2003). In brief, the results indicate that the MISC offers generally good interrater reliability in capturing the global dimensions or composite indicators of interactions in MI sessions. Ratings of more specific behaviors are less reliable. In describing the strength of the ICCs we draw on the categorization system proposed by Cicchetti (1994) for evaluating the usefulness of ICCs in clinical instruments: below $.40=$ poor, .40-.59= fair, .60-.74= good and .75$1.00=$ excellent. ICCs are provided below for all the composite variables as well as each of the frequency count variables that are used individually in analyses.

\section{Measures}

Counselor adherence was assessed with a composite variable of subjective global ratings as well as frequency counts of specific MI-consistent and MI-inconsistent behaviors. The frequency counts were also combined to form two composite variables (MI-consistent and MI-inconsistent frequency). Four variables were used to index client behavior: a subjective global rating of client functioning, a subjective global rating of the quality of the interaction between counselor and client, and the frequency of statements constituting change talk and resist-change talk. Each of these is detailed below.

Counselor MI-global rating. This rating consists of an average of six qualitative dimensions (scored on a scale of 1-7) of theoretical importance to MI: therapist's demonstration of acceptance $(1=$ low, $7=$ high $)$, egalitarianism $(1=$ authoritarian, $7=$ egalitarian $)$, warmth $(1=$ cold, $7=$ warm), genuineness, empathy, and overall adherence to the spirit of motivational interviewing $(1=$ low, $7=$ high $)$. Interrater reliability for acceptance and warmth were "poor" and "fair" (ICCs $=.39$ and .48 , respectively), while ratings for egalitarianism and genuineness were "good" (ICCs $=.68$ and .69 , respectively) and ratings for empathy and spirit were "excellent" (ICCs $=.77$ and .79 , respectively). Interrater reliability for the composite was judged as "excellent" ( ICC $=.77)$. 
Counselor MI-consistent response count. This composite variable sums the number of times the therapist engages in behaviors that are consistent with MI: advise with permission $(\mathrm{ICC}=-.04)$, affirm $(\mathrm{ICC}=.38)$, emphasize control $(\mathrm{ICC}=1.00)$, ask an open question $(\mathrm{ICC}=.55)$, reflect $(\mathrm{ICC}=.72$ for repeat; .82 for rephrase; .78 for paraphrase; .24 for summarize), reframe $(\mathrm{ICC}=.79)$, and support $(\mathrm{ICC}=.65)$. Interrater reliability for the composite was "excellent" $(\mathrm{ICC}=.81)$.

Counselor MI-inconsistent response count. This composite variable sums the number of times the therapists demonstrate six behaviors that are inconsistent with the MI approach: advise without permission $(\mathrm{ICC}=.03)$, confront $(\mathrm{ICC}=.00)$, direct $(\mathrm{ICC}=.57)$, raise concern without permission $(\mathrm{ICC}=.21)$, and warn $(\mathrm{ICC}=.37)$. Interrater reliability for the composite was "fair" ( $\mathrm{ICC}=.51)$.

Client MI global rating of within-session functioning. This rating consists of an average of four qualitative dimensions (scored on a scale of $1-7 ; 1=$ low, $7=$ high) that indicate the therapist elicited client responses that are of theoretical importance to MI: expressing affect, cooperating, self-disclosing, and being engaged. Interrater reliability for this composite was "good" (ICC = .74).

Client change talk count. These are statements the client makes that are favorable to changing the behavior of interest including statements acknowledging the need for change (problem recognition, expressing concern) and expressing desire, intention, or optimism for change. Interrater reliability for this variable was "excellent" $(\mathrm{ICC}=.78)$.

Client resist-change talk count. These are statements the client makes that are opposed to or resistant to changing the behavior of interest including arguing, interrupting, negating, or not following. Interrater reliability for this variable was "fair" $(\mathrm{ICC}=.53)$.

Counselor-client interaction rating. This rating consists of an average of two qualitative dimensions (scored on a scale of $1-7 ; 1=$ low, $7=$ high) of how the counselor and client work together during the session in ways that are consistent with MI: collaboration (forming a partnership for change) and benefit (client moves towards positive change during the session). Interrater reliability for this composite score variable was "good" $(\mathrm{ICC}=.63)$.

\section{Statistical analyses}

All statistical analysis on the data was performed using the SAS Software System (SAS Institute Inc, Cary, NC). A 5\% level of significance was used for all models. The MISC variables were summarized using means, ranges and standard deviations. Mixed models were used to examine whether adherence to MI principles predicted each of three separate client behaviors: client within-session functioning, counselor-client interaction, and client statements related to behavior change (i.e. "change talk" and "resist-change talk"). Adherence to MI principles was indexed in two ways: in one model the subjective counselor MI global rating was used as the predictor variable, while in a second model, more objective counselor MI-consistent and counselor MI-inconsistent response counts were used as predictors. The mixed model was chosen because of the hierarchical nature of the data in which multiple sessions were conducted by each of the four counselors (i.e. the counseling sessions were not independent because of the clustering at the level of counselor). A compound symmetric covariance matrix was assumed 
Table 1. Ranges, means, and standard deviations of MISC variables

\begin{tabular}{lcrr}
\hline Measure & Range & Mean & \multicolumn{1}{c}{$S D$} \\
\hline Client behavior variables & & & \\
Client MI global rating & $2.0-6.5$ & 4.82 & 1.20 \\
Interaction global rating & $2.5-6.0$ & 4.70 & 0.89 \\
Client change talk counts & $1-37$ & 12.02 & 6.88 \\
Client resist-change talk counts & $0-16$ & 3.90 & 3.04 \\
Counselor MI global adherence & $1.8-6.2$ & 4.09 & 1.28 \\
Counselor MI-consistent count & $8-59$ & 28.99 & 11.71 \\
Advise with permission & $0-3$ & .53 & .85 \\
Affirm & $0-9$ & 2.88 & 2.05 \\
Emphasize control & $0-9$ & 1.07 & 1.40 \\
Open question & $1-25$ & 5.79 & 3.60 \\
Reflect & $4-37$ & 17.55 & 7.00 \\
Reframe & $0-4$ & .07 & .45 \\
Support & $0-6$ & 1.09 & 1.41 \\
Counselor MI-inconsistent count & $2-28$ & 10.91 & 5.12 \\
Advise without permission & $0-8$ & 4.22 & 1.95 \\
Confront & $0-4$ & .28 & .70 \\
Direct & $0-16$ & 5.86 & 3.68 \\
Raise concern without permission & $0-3$ & .15 & .47 \\
Warn & $0-7$ & .40 & 1.15 \\
\hline
\end{tabular}

to account for the correlation resulting from the counselor effect. Similar mixed models were also used to further examine whether individual MI-consistent and MI-inconsistent response variables predicted each of the three separate client behavior variables.

\section{Results}

Summary statistics for all of the variables used in analyses are presented in Table 1. One good descriptive indicator of counselor adherence to MI in this study was provided by the global adherence variable. As can be seen in Table 1, the mean for global adherence was 4.09 and the range of scores went from very low adherence (1.8) to expert (6.2). The mean was below the proficiency threshold of 5.0 that is suggested by the MISC; however, the range of scores indicated that there were counseling sessions in which there was very low adherence and other sessions in which there was expert level adherence. This pattern was expected because although counselors were trained in MI and were qualified counselors, for the purposes of the original study it was not critical to ensure consistency in meeting any proficiency criterion with respect to MI.

We also examined whether there were significant mean differences between counselors in global MI adherence ratings. Results of a linear regression model indicated there was a significant counselor effect, $F(3,82)=69.71, p<.001$. The means for each counselor were $3.86,5.19,2.70$, and 4.69. Counselor was used as a covariate in all models to allow us to focus on differences in outcome related to MI behaviors rather than other counselor traits.

Results of the analyses examining the association between the global and composite indicators of adherence to MI principles and the three client behavior variables are presented 
Table 2. Results of mixed modeling examining associations between MI-Consistent counseling and client functioning, counselor-client interactions, and client responses regarding change

\begin{tabular}{|c|c|c|c|c|c|c|c|c|}
\hline \multirow[b]{3}{*}{ Predictor variable } & \multicolumn{8}{|c|}{ MISC client behavior variable } \\
\hline & \multicolumn{2}{|c|}{$\begin{array}{l}\text { Client global } \\
\text { functioning }\end{array}$} & \multicolumn{2}{|c|}{ Interaction global } & \multicolumn{2}{|c|}{ Change talk } & \multicolumn{2}{|c|}{$\begin{array}{l}\text { Resist-change } \\
\text { talk }\end{array}$} \\
\hline & Estimate & SE & Estimate & SE & Estimate & SE & Estimate & SE \\
\hline \multicolumn{9}{|l|}{ Model 1} \\
\hline Counselor MI global $^{\mathrm{a}}$ & $.567^{* * *}$ & .121 & $.688^{* * *}$ & .071 & $3.265^{* * *}$ & .856 & .396 & .382 \\
\hline \multicolumn{9}{|l|}{ Model 2} \\
\hline Counselor MI-consistent $\mathrm{t}^{\mathrm{b}}$ & $.027^{*}$ & 011 & $.043^{* * *}$ & .007 & $.357^{* * *}$ & .069 & .013 & .034 \\
\hline Counselor MI-inconsistent $^{\mathrm{b}}$ & -.031 & .024 & -.020 & .016 & -.236 & .145 & -.026 & .070 \\
\hline
\end{tabular}

Note . All models are adjusted for counselor effects. Estimate $=$ Parameter Estimate; $\mathrm{SE}=$ Standard Error

${ }^{\mathrm{a}}$ In all models $d f=1,81$.

${ }^{\mathrm{b}}$ In all models $d f=1,80 .{ }^{*} p<.05 .^{* * *} p<.001$

Table 3. Results of mixed modeling examining associations between individual MI-consistent and MI-inconsistent response variables and client functioning, counselor-client interactions, and client responses regarding change

\begin{tabular}{|c|c|c|c|c|c|c|c|c|}
\hline \multirow[b]{3}{*}{ Predictor variable } & \multicolumn{8}{|c|}{ MISC client behavior variable } \\
\hline & \multicolumn{2}{|c|}{$\begin{array}{l}\text { Client global } \\
\text { functioning }\end{array}$} & \multicolumn{2}{|c|}{ Interaction global } & \multicolumn{2}{|c|}{ Change talk } & \multicolumn{2}{|c|}{$\begin{array}{l}\text { Resist-change } \\
\text { talk }\end{array}$} \\
\hline & Estimate & SE & Estimate & SE & Estimate & SE & Estimate & SE \\
\hline \multicolumn{9}{|l|}{ Counselor MI-consistent } \\
\hline Advise with permission & .028 & .138 & .084 & .105 & -.917 & .939 & -.592 & .385 \\
\hline Affirm & $.113^{*}$ & .056 & $.098^{*}$ & .042 & .606 & .383 & -.195 & .160 \\
\hline Emphasize control & .063 & .079 & .020 & .060 & .376 & .537 & $-.462^{*}$ & .217 \\
\hline Open question & .057 & .032 & $.094^{* * *}$ & .023 & $.377^{\dagger}$ & .221 & .018 & .093 \\
\hline Reflect & .023 & .017 & $.050^{* * *}$ & .012 & $.565^{* * *}$ & .102 & .055 & .049 \\
\hline Reframe & .013 & .206 & .053 & .157 & $5.349^{* * *}$ & 1.271 & $2.056^{* * *}$ & .533 \\
\hline Support & $.151^{\dagger}$ & .077 & $.118^{*}$ & .059 & .481 & .534 & .098 & .222 \\
\hline \multicolumn{9}{|l|}{ Counselor MI-inconsistent } \\
\hline Advise without permission & $-.093^{\dagger}$ & .048 & -.033 & .037 & $-.662^{*}$ & .323 & -.045 & .138 \\
\hline Confront & -.112 & .144 & $-.380^{* * *}$ & .102 & -.705 & .982 & .234 & .406 \\
\hline Direct & -.006 & .033 & .007 & .025 & -.099 & .225 & -.072 & .093 \\
\hline $\begin{array}{l}\text { Raise concern without } \\
\text { permission }\end{array}$ & .321 & .210 & .138 & .162 & $3.076^{*}$ & 1.414 & .574 & .598 \\
\hline Warn & -.150 & .102 & $-.200^{*}$ & .076 & $-1.306^{\dagger}$ & .692 & .178 & .292 \\
\hline
\end{tabular}

Note. All models are adjusted for counselor effects. In all models $d f=1,81$. Estimate $=$ Parameter Estimate; $\mathrm{SE}=$ Standard Error.

${ }^{\dagger} p<.07 .{ }^{*} p<.05 .{ }^{* * *} p<.001$.

in Table 2. Analyses examining the association between each individual MI-consistent and inconsistent counselor behavior that make up the composite indicators and each of the three client behavior variables are presented in Table 3 . 


\section{Client global functioning}

The client global rating (average of affect, cooperation, self-disclosure, and engagement) was used as the index of client functioning. As can be seen in Table 2, results of mixed modeling analyses indicated that MI global ratings and MI-consistent frequency were significantly positively associated with global client functioning ratings, whereas the frequency of MIinconsistent behaviors was not related to client functioning.

With respect to the association between each MI-consistent and MI-inconsistent behavior and each client behavior variable (displayed in Table 3), separate models revealed that client global rating was only significantly positively associated with the mean frequency of affirm responses, although there was a positive association with support $(p<.053)$, and a negative association with advise without permission that approached significance $(p<.053$ and $p<.055$, respectively).

\section{Counselor-client interactions}

The counselor-client interaction rating (average of "collaboration" and "benefit") was used as the dependent variable. Results, also displayed in Table 2, indicated counselor MI global adherence and MI-consistent frequency were significantly positively related to the interaction rating; however, MI-inconsistent frequency was not significantly related to the interaction rating.

With respect to the individual MI behaviors displayed in Table 3, the interaction rating was significantly positively associated with affirm, open question, reflect, and support. The interaction rating was negatively related to confront and warn.

\section{"Change talk" and "resist-change talk"}

The client change talk frequencies and client resist-change talk frequencies were used as dependent variables in separate sets of models (displayed in Table 2). The models predicting change talk frequency followed the same pattern as for the other dependent variables examined. MI global adherence and MI-consistent frequency (but not MI-inconsistent frequency) were significant positive predictors of change talk. The results for client resist-change talk frequency were the exception to the general pattern with no significant predictors emerging.

With respect to the individual MI behaviors displayed in Table 3, client change talk was significantly positively related to reflective statements, reframing statements, and, unexpectedly, raising concern without permission. Change talk was significantly negatively related to giving advice without permission. Also, a negative association was observed between warn and change talk that approached significance $(p<.063)$. With regard to client resistchange talk, results indicated a significant negative association with emphasize control and an unexpected significant positive association with reframe.

\section{Discussion}

The main purpose of this study was to examine whether adherence to MI principles would be associated with more productive within-session client behavior including more change talk 
and less resistant statements. Results revealed that both subjectively rated global MI adherence and the frequency of overall MI-consistent behavior predicted better client functioning (i.e. expression of affect, cooperation, disclosure, and engagement), better counselor-client interaction (i.e. collaboration and benefit), and more change talk. Results thus generally supported our hypothesis that adherence to MI principles would be associated with better within-session client behavior, including a greater frequency of change talk. Contrary to expectations, the overall frequency of MI-inconsistent behavior was not associated with client functioning, and resist-change talk was not predicted by either the subjective rating of global MI adherence or the overall frequency of MI-consistent or MI-inconsistent behaviors.

These results are generally consistent with the findings of Miller et al. (1993) who found that an MI-consistent style was related to more functional client behaviors during a discussion about client drinking behavior. In the Miller study an MI style, compared to a directive style, yielded less client arguing, interrupting, denying, and off-task behaviors and more acknowledging of problems (i.e. change talk). The present study extends these findings by demonstrating that greater adherence to an MI style not only elicits more change talk but also leads to improvement in other indicators of positive within-session client functioning (i.e. expression of affect, cooperation, disclosure and engagement) and client-counselor interactions (i.e. collaboration and benefit).

One notable difference between the results of the present study and the Miller et al. (1993) study is that we found no associations between MI-inconsistent behaviors and our client behavior variables. One possible explanation for this difference is that the Miller study contrasted MI with a confrontational approach that is the antithesis of MI, whereas in the present study we relied on natural variation in adherence to MI by counselors who were all attempting to adhere to MI principles. Although less use of MI-consistent counselor behaviors may reduce the likelihood of client change talk, more extreme engagement in the proscribed MI-inconsistent behaviors may be necessary to produce significant negative (resist-change) client responses. An alternative explanation is that participants in this study were more ready to change, given that they had made the decision to enroll in a quit smoking program. This may have increased the likelihood of more positive interactions. Yet another explanation is that lower reliability of the MI-inconsistent behavior reduced the likelihood of detecting significant associations.

Analyses examining associations between individual MI-consistent and inconsistent behaviors and the client behavior variables produced a number of significant associations. Because of the low reliability of the counselor behavior frequency variables and because of the high number of associations we examined, these findings should be interpreted cautiously. Nevertheless, only two of the twelve MI-consistent and MI-inconsistent counselor behaviors (advise with permission and direct) had no significant associations with any of the client behavior variables (client functioning, client-counselor interactions, change talk, and resistchange talk). All but two of the associations (which are discussed below) were in the expected direction.

With respect to predictors of change talk, it is notable that reflect and reframe significantly correlated with change talk. This is almost identical to Miller et al.'s (1993) report that therapist "listening" and "restructuring" was highly correlated with "positive" verbal responses from the client such as agreeing with the therapist, or expressing concern, determination, or optimism. A surprising result, not consistent with MI principles, was that raising concern without permission was associated with greater change talk. One possible explanation for 
this result is that it reflects a process of counselors responding to clients rather than the other way around. When clients are talking frequently about changing, perhaps including their plans for change, counselors may be more likely to express any concerns they have than when clients are not really talking about change. Whatever the explanation for this result, this possibility highlights an important limitation of this study. Coding of behavior was not based on the sequence of counselor statements and client responses. All of the findings are correlational and cannot be taken as proof that particular MI-consistent behaviors lead to particular client responses. Future research may be strengthened by coding interactions sequentially.

With respect to resist-change talk, we observed a number of discrepancies from the findings of Miller et al. (1993). Perhaps most notably, confront was not predictive of resist-change talk in the present study. In fact, none of the MI-inconsistent behaviors were predictive of resist-change talk. Low reliability of both the MI-inconsistent behavior variables and the resist-change talk variable may be the most likely explanation for this discrepancy. Another surprising result was that reframe (which we noted above was associated with more change talk) also had a significant positive association with resist-change talk. Although this might be a function of counselor's using reframes when clients resist, this may also suggest that a better understanding of the circumstances under which reframing produces change talk rather than resistance is needed. Perhaps reframing is sometimes perceived as helpful and sometimes perceived as a sign of lack of empathy. The only other therapist behavior related to resist-change talk was emphasize control which, consistent with Miller and Rollnick's (2002) suggested use of this strategy, was associated with less resistance.

In summary, the individual behavior analyses suggest that to increase change talk and minimize resistance one should focus specifically on using more reflections, emphasizing client autonomy, and avoiding giving advice without permission. Greater understanding is needed of the use of reframing and raising concern without permission. Given recent promising evidence of the association between change talk and drug use outcomes (Amrhein et al., 2003), it is particularly useful to have evidence of how MI may elicit change talk. Amrhein et al.'s results also point to future directions for this line of research. Amrhein et al. found that a particular form of change talk concerning commitment to change or maintain drug use behavior was most predictive of drug use outcomes. More specifically, it was the strength of commitment expressed rather than the frequency of expression that was most predictive. Also, commitment expressed in the middle and at the end of the session was most predictive. Future studies could improve on the methods of the present study by using a revised version of the MISC that captures the different types of change talk and could perhaps explore which counselor behaviors are associated with commitment strength in the middle and end of sessions.

While change talk is theoretically important, and has some empirical support as an important correlate of positive therapeutic outcomes (Amrhein et al., 2003), the counselor-client interaction variable used in this study represented a more direct indicator of potentially positive therapeutic outcomes. This variable included observer-rated collaboration and apparent client benefit during the session. Interestingly, reflections were also correlated with this client variable as was the use of open-ended questions, supportive statements, client affirmations, and less confronting and warning. Miller et al. (1993) found that confronting was the only therapist behavior predictive of (poor) drug use outcomes at follow-up. The present study's within-session results also suggest that avoiding confrontation is important, but point to a 
number of other behaviors that may be particularly important for counselors to use to improve client benefit. Indeed, the behaviors that were predictive of positive interactions in this study encompass the majority of key MI consistent behaviors. Further research is needed to confirm these preliminary findings.

The overall pattern of findings not only provides support for MI principles but also supports the validity of the MISC. This is the first study we are aware of to report associations between the various elements of the MISC. Associations between counselor ratings and client behavior ratings were generally as expected. Similar findings for the subjective global ratings of the counselor and the more objective overall MI-consistent behavior frequency provide evidence for the validity of both approaches in assessing counselor behavior. On a more negative note, the reliability of MI-inconsistent behavior measures was uniformly poor and may have contributed to the generally fewer significant associations between counselor MI-inconsistent behavior and client behavior. The same may have been true for the measure of resist-change talk, which had only fair reliability and had relatively few associations with counselor behaviors. It is also worth noting that use of the MISC requires considerable expertise, training, and time. For example, only the first 20 minutes of sessions were coded in this study to reduce the time and cost burden. This leaves the possibility that important events that occurred late in the session were not captured. These limitations notwithstanding, the MISC clearly represents a valuable tool for assessing counselor behavior and further research to establish reliability and validity and to improve its utility is warranted.

In addition to reliability concerns, another important limitation of this study was that MI adherence and client behavior variables were not independently rated. It is possible that raters were more inclined to see positive client behaviors in sessions that they noted greater MI adherence. However, our confidence in the findings is enhanced by the similar results obtained with the subjective behavior ratings (client global functioning and interaction global) and the more objective behavior count ratings (change talk and resist-change talk). In addition, it is unlikely that raters would have had biases sufficiently nuanced to influence which specific MI prescribed and proscribed behaviors would predict client behaviors.

Another limitation of the study was that we lacked the ability to effectively examine the association between MI adherence and change in the targeted behavior of smoking cessation. Evidence of which specific MI prescribed or proscribed behaviors (or combination of behaviors) lead to behavior change outcomes is a pressing need for future research. With regard to generalizability of the findings, it is important to recognize that this study was in the context of smoking cessation intervention conducted for research purposes. Counseling was relatively brief and followed a semi-structured format. Participants were also all African American and relatively heavy smokers. The extent to which the present results will generalize to other groups and other counseling settings is unknown.

Despite these limitations, the study had a number of strengths, including the relatively unusual demographic characteristics of the sample and the fact that it is one of the few studies of the use of MI for smoking cessation. Another strength of the study was that it capitalized on the fact that there were no counselor fidelity control procedures. A typical clinical trial testing MI is likely to have a great deal less variability in therapist adherence to MI principles than the present study, making it far more difficult to detect effects of adherence.

In conclusion, the results make an important contribution to the MI literature by providing general support for the value of adhering to the key principles that define MI. MI trainers and 
practitioners may benefit from more research on the MI process to deepen our understanding of how and why MI helps clients to change.

\section{Acknowledgements}

We thank Dr. Theresa Moyers and Tim Martin who led the effort to code the audiotapes for this project, as well as Ken Resnicow, Ph.D. for his guidance with this research.

This research was funded by grants from the National Cancer Institute (R01 CA77856, K07 CA87714 and K07 CA90334). Glaxo-Wellcome Inc. provided medication for the original study from which these data are drawn.

\section{References}

Ahluwalia, J. S., Harris, K. J., Catley, D., Okuyemi, K. S. and Mayo, M. S. (2002). Sustained-release bupropion for smoking cessation in African Americans: a randomized controlled trial. Journal of American Medical Association, 288, 468-474.

Amrhein, P. C., Miller, W. R., Yahne, C. E., Palmer, M. and Fulcher, L. (2003). Client commitment language during motivational interviewing predicts drug use outcomes. Journal of Consulting and Clinical Psychology, 71, 862-878.

Brown, J. M. and Miller, W. R. (1993). Impact of motivational interviewing on participation and outcome in residential alcoholism treatment. Psychology of Addictive Behaviors, 7, 211218.

Burke, B. L., Arkowitz, H. and Dunn, C. (2002). The efficacy of motivational interviewing and its adaptations: what we know so far. In W. R. Miller and S. Rollnick (Eds.), Motivational Interviewing: preparing people for change (2nd ed.). New York: Guilford Press.

Cicchetti, D. V. (1994). Guidelines, criteria and rules of thumb for evaluating normed and standardized assessment instruments in psychology. Psychological Assessment, 6, 284-290.

Gentilello, L. M., Rivara, F. P., Donovan, D. M., Jurkovich, G. J., Daranciang, E., Dunn, C. W. et al. (1999). Alcohol interventions in a trauma center as a means of reducing the risk of injury recurrence. Annals of Surgery, 230, 473-483.

Handmaker, N. S., Miller, W. R. and Manicke, M. (1999). Findings of a pilot study of motivational interviewing with pregnant drinkers. Journal of Studies on Alcohol, 60, 285-287.

Miller, W. R. and Baca, L. M. (1983). Two-year follow-up of bibliotherapy and therapist-directed controlled drinking training for problem drinkers. Behavior Therapy, 14, 441-448.

Miller, W. R., Benefield, G. and Tonigan, J. S. (1993). Enhancing motivation for change in problem drinking: a controlled comparison of two therapist styles. Journal of Consulting and Clinical Psychology, 61, 455-461.

Miller, W. R. and Mount, K. (2001). A small study of training in motivational interviewing: does one workshop change clinician and client behavior? Behavioural and Cognitive Psychotherapy, 29, 457-471.

Miller, W. R. and Rollnick, S. (1991). Motivational Interviewing: preparing people to change addictive behavior. New York: Guilford Press.

Miller, W. R. and Rollnick, S. (2002). Motivational Interviewing: preparing people for change (2nd ed.). New York: Guilford Press.

Miller, W. R., Taylor, C. A. and West, J. C. (1980). Focused versus broad-spectrum behavior therapy for problem drinkers. Journal of Consulting and Clinical Psychology, 48, 590-601.

Moyers, T., Martin, T., Catley, D., Harris, K. J. and Ahluwalia, J. S. (2003). Assessing the integrity of motivational interviewing interventions: reliability of the Motivational Interviewing Skills Code. Behavioural and Cognitive Psychotherapy, 31, 177-184. 
Patterson, G. R. and Forgatch, M. S. (1985). Therapist behavior as a determinant for client noncompliance: a paradox for the behavior modifier. Journal of Consulting and Clinical Psychology, $53,846-851$.

Resnicow, K., Diiorio, C., Soet, J. E., Borelli, B., Hecht, J. and Ernst, D. (2002). Motivational Interviewing in health promotion: it sounds like something is changing. Health Psychology, 21, 444-451.

Resnicow, K., Jackson, A., Braithwaite, R., Diiorio, C., Blisset, D., Perisamy, S. et al. (2002). Healthy body/healthy spirit: design and evaluation of a church-based nutrition and physical activity intervention using motivational interviewing. Health Education Research, 17, 562-573.

Tappin, D. M., McKay, C., McIntyre, D., Gilmour, G. W., Cowan, S., Crawford, F. et al. (2000). A practical instrument to document the process of motivational interviewing. Behavioural and Cognitive Psychotherapy, 28, 17-32.

Valle, S. K. (1981). Interpersonal functioning of alcoholism counselors and treatment outcome. Journal of Studies on Alcohol, 42, 783-790.

Wagner, C. C. and Sanchez, F. P. (2002). The role of values in Motivational Interviewing. In W. R. Miller and S. Rollnick (Eds.). Motivational Interviewing: preparing people for change (2nd ed.). New York: Guilford Press. 